K.A. van der Hucht, G. Koenigsberger \& P.R.J. Eenens, eds.

\title{
The fate of superbubbles in dwarf starburst galaxies
}

\author{
Sergey A. Silich ${ }^{1}$ and Guillermo Tenorio-Tagle ${ }^{2}$ \\ ${ }^{1}$ Main Astronomical Observatory, National Academy of Sciences, \\ 252650 Kiev, Golosiiv, Ukraine \\ ${ }^{2}$ INAOE, Apartado Postal 51, Tonantzintla, 72000 Puebla, México
}

\begin{abstract}
D calculations of the evolution of the remnants generated by the strong mechanical energy produced by a central starburst in dwarf galaxies are presented. We show how the extended $\mathrm{H}$ I halos inhibit a galactic wind and thus the exit of metals from these galaxies.
\end{abstract}

\section{Introduction}

It is a common believe that the activity of violent starbursts (SB)s produces, particularly in low mass galaxies, powerful galactic winds causing the loss of processed elements, and is even able to strip galaxies of their entire ISM (De Young \& Heckman 1994). Most estimates however, extrapolate from the results obtained for the most energetic SBs. Also, they do not include the contribution of the dark matter component, nor the possible impact that an extended low density halo may have on the evolution of superbubbles. Our calculaltions (Silich \& Tenorio-Tagle 1998) take into consideration all of these issues and assume a realistic energy-input rate in agreement with the observations of dwarf galaxies.

\section{Input model}

We model a galaxy ISM with three different isothermal components: a thin neutral disk, a thick ionized disk, and an extended turbulent halo. Both the radial density gradients and the smooth transition from a slow rotating disk to a non-rotating spherical halo are included. The contributions to the gravitational field from a stellar and a dark matter component are here approximated with a King model and a distribution inversely proportional to the square of the radius, respectively. The calculations consider $10^{8-10} \mathrm{M}_{\odot}$ galaxies with different extensions of the dark matter component and different velocity dispersions of 60 to $120 \mathrm{~km} \mathrm{~s}^{-1}$ of the extended halos. The mass of the gaseous component has been assumed to equal $10 \%$ of the total galaxy mass in all models. The calculations also include thermal evaporation, and mass ejection as a result of SN explosions. An energy input rate $L_{*}=10^{40-41} \mathrm{erg} \mathrm{s}^{-1}$ (Marlowe et al. 1995) was assumed to be constant during the starburst phase $t_{\mathrm{SB}}=40 \mathrm{Myr}$.

\section{Discussion and conclusions}

Figure 1 compares the superbubble largest speed as a function of position (time) with the galaxy escape velocity. Three phases are evident in the plot: (1) the 


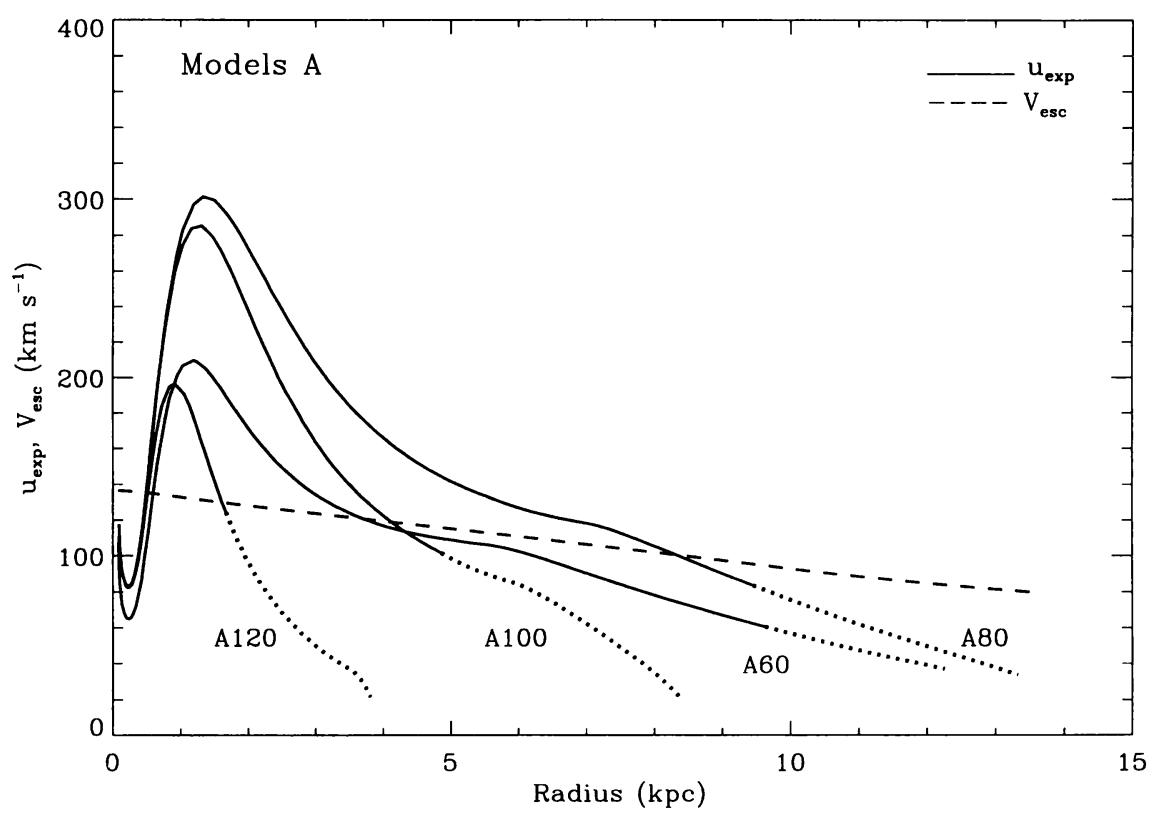

Figure 1. Evolving superbubbles. The largest expansion speed (solid lines) compared with the galaxy escape velocity (dashed line).

initial deceleration; (2) the acceleration promoted by blowout; and (3) a final steady deceleration caused by the sweeping of the extended halo matter. The last leads to velocities well below the galaxy escape speed, as detected in the sample of Marlowe et al. (1995). The estimated mechanical luminosity that could allow for a true galactic wind is much larger than the values derived from detailed observations. We thus conclude that superbubbles in well studied dwarfs are most likely observed in the post-blowout period, i.e., after the superbubble has burst its energy into the extended $\mathrm{H}$ I halo. And consequently, these galaxies are able to retain the metals released during the life-time of the SB and clearly all of their ISM.

Acknowledgments. SAS acknowledges an IAU grant, and a Royal Society grant for joint projects with the former Soviet Union.

\section{References}

De Young, D.S., Heckman, T.M. 1994, ApJ 431, 598

Marlowe, A.T., Heckman, T.M., Wyse, R.F.G., Schommer, R. 1995, ApJ 438, 563

Silich, S.A., Tenorio-Tagle, G. 1998, MNRAS 299, 249 